Lauren Akesson $\quad$ ORCID iD: 0000-0002-7439-612X

Adam Bournazos $\quad$ ORCID iD: 0000-0002-6464-4548

Zornitza Stark ORCID iD: 0000-0001-8640-1371

Sandra Cooper ORCID iD: 0000-0002-7314-5097

\title{
Rapid exome sequencing and adjunct RNA studies confirm pathogenicity of a novel homozygous $A S N S$ splicing variant in a critically ill neonate
}

\author{
*Lauren S. Akesson ${ }^{1,2,3}$ \\ *Adam Bournazos ${ }^{4,5}$ \\ *Andrew Fennell ${ }^{3,6}$ \\ Emma I. Krzesinski ${ }^{3,6}$
}

Kenneth $\operatorname{Tan}^{6,7}$

Amanda Springer 3,6

Katherine Rose $\mathrm{e}^{3,6}$

Ilias Goranitis 8,9

David Francis $^{1}$

Crystle Lee ${ }^{1}$

Fathimath Faiz ${ }^{10}$

This is the author manuscript accepted for publication and undergone full peer review but has not been through the copyediting, typesetting, pagination and proofreading process, which may lead to differences between this version and the Version of Record. Please cite this article as doi: 10.1002/humu.24101.

This article is protected by copyright. All rights reserved. 
Mark R. Davis ${ }^{10}$

John Christodoulou ${ }^{1,2,9,11}$

Sebastian Lunke ${ }^{1,9,12}$

**Zornitza Stark ${ }^{1,2,9}$

**Matthew F. Hunter ${ }^{3,6} * *$ Sandra T. Cooper ${ }^{4,5,13}$

*Lauren S. Akesson, Adam Bournazos, and Andrew Fennell contributed equally as joint first authors.

**Sandra T. Cooper, Matthew F. Hunter, and Zornitza Stark contributed equally as joint senior authors.

1. Victorian Clinical Genetics Services, Melbourne, Australia

2. Department of Paediatrics, University of Melbourne, Melbourne, Australia

3. Monash Genetics, Monash Health, Melbourne, Australia

4. Kids Neuroscience Centre, Children's Hospital at Westmead, Sydney, Australia

5. University of Sydney, Sydney, Australia

6. Department of Paediatrics, Monash University, Melbourne, Australia

7. Monash Newborn, Monash Health, Melbourne, Australia

8. Melbourne School of Population and Global Health, University of Melbourne, Melbourne, Australia

9. Australian Genomics Health Alliance, Australia

10. Department of Diagnostic Genomics, PathWest Laboratory Medicine, Perth, Australia

This article is protected by copyright. All rights reserved. 
11. Brain and Mitochondrial Research Group, Murdoch Children's Research Institute, Melbourne, Australia

12. Department of Clinical Pathology, University of Melbourne, Melbourne, Australia

13. Children's Medical Research Institute, Sydney, Australia

\section{Corresponding author:}

Associate Professor Zornitza Stark

Victorian Clinical Genetics Services

Murdoch Children's Research Institute

Flemington Road

Parkville VIC 3052

Australia

Email: Zornitza.Stark@vcgs.org.au

Phone: +61383416368

\section{Grant numbers}

The Australian Genomics Acute Care study was funded by the Australian National Health and Medical Research Council Targeted Call for Research (GNT1113531). Research conducted at the Murdoch Children's Research Institute was supported by the Victorian Government's Operational Infrastructure Support Program. STC and AB are supported by a National Health and Medical Research Council of Australia Senior Research Fellowship (APP1136197 and APP1080587) and Rapid Splicing studies

This article is protected by copyright. All rights reserved. 
were supported by a Sydney Health Partners Medical Research Future Foundation Rapid Applied Research Translation grant. AB is supported by a University of Sydney Research Training Scholarship.

\title{
Conflict of Interest Statement
}

Professor Sandra Cooper is director of Frontier Genomics Pty Ltd (Australia). Frontier Genomics has not traded (as of October 2019). Frontier Genomics Pty Ltd (Australia) has no existing financial relationships that will benefit from publication of these data. The remaining co-authors do not have any relationships, financial or otherwise, that may result in a perceived conflict of interest.

\begin{abstract}
Rapid genomic diagnosis programs are transforming rare disease diagnosis in acute pediatrics. A ventilated newborn with cerebellar hypoplasia underwent rapid exome sequencing (75 hours), identifying a novel homozygous ASNS splice-site variant (NM_133436.3:c.1476+1G>A) of uncertain significance. Rapid ASNS splicing studies using blood-derived mRNA from the family trio confirmed a consistent pattern of abnormal splicing induced by the variant (cryptic 5' splice-site or exon 12 skipping) with absence of normal ASNS splicing in the proband. Splicing studies reported within 10 days led to reclassification of c. $1476+1 \mathrm{G}>\mathrm{A}$ as pathogenic at age 27 days. Intensive care was redirected towards palliation. Cost analyses for the neonate and his undiagnosed, similarly affected deceased sibling, demonstrate that early diagnosis reduced hospitalization costs by AU $\$ 100,828$. We highlight diagnostic benefits of adjunct RNA testing to confirm pathogenicity of splicing variants identified via rapid genomic testing pipelines for precision and preventative medicine.
\end{abstract}

This article is protected by copyright. All rights reserved. 


\section{Graphical Abstract}

We present rapid RNA studies that diagnosed a seriously ill newborn with Asparagine Synthetase Deficiency after a homozygous ASNS splicing variant was detected by ultra-rapid exome sequencing.

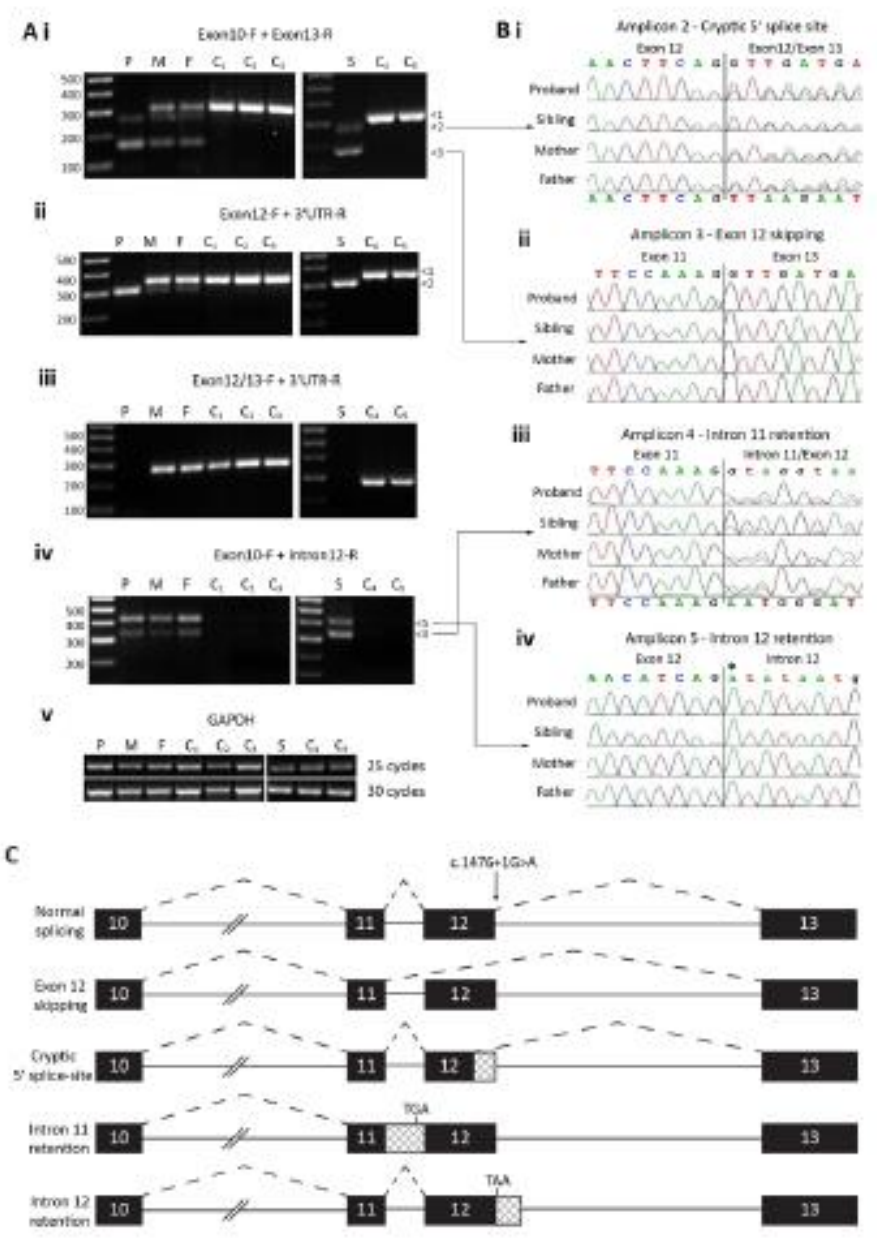

\section{KEYWORDS}

Asparagine synthetase deficiency

ASNS

Exome sequencing

mRNA splicing analysis

Rapid genomic diagnosis program

This article is protected by copyright. All rights reserved. 


\section{MAIN TEXT}

Rapid genomic diagnosis programs in neonatal and pediatric intensive care are transforming clinical practice by diagnosing monogenic disorders in less than 20 hours (Clark et al., 2019). However, assessment of variants of uncertain significance identified in phenotypically concordant genes remains challenging, as supportive functional validation studies such as mRNA and functional protein analyses are not typically available within clinically meaningful timeframes. We present a seriously ill newborn who was diagnosed with asparagine synthetase deficiency as part of a rapid genomic diagnosis program, where the pathogenicity of a splicing variant of uncertain significance was confirmed via an integrated pipeline of rapid mRNA analyses. This case demonstrates the clinical and cost benefits of rapid mRNA analyses as part of a rapid genomic diagnosis program in pediatric acute care. A timeline for the diagnostic investigations undertaken in this case, including research-based mRNA splicing studies, is provided in Figure 1 part C. Additional information on the methodologies is given in the Supplementary Information, available online.

The proband (II:6, Figure 1A-B) was a male neonate born at 36 weeks' gestation to non-consanguineous parents (I:1 and I:2) of South Sudanese ethnicity, following a pregnancy complicated by antenatal detection of microcephaly and suspected pontocerebellar hypoplasia. The family had previously received genetic counselling in 2011 following the death of a male sibling (II:2) at age 8 weeks. The sibling had a similar antenatal course to the proband and was ventilator-dependent from birth. He had significant microcephaly with a head circumference Z-score of -4.2 with otherwise normal growth parameters. In the absence of clinical improvement, the family elected to redirect the sibling's care towards palliation and symptomatic 
management, and the infant died at age 8 weeks. Post-mortem examination was declined. A suspected diagnosis of pontocerebellar hypoplasia type 4 was based on brain magnetic resonance imaging (MRI) findings (Figure 1A). Chromosomal microarray was normal and no further genetic testing was offered. The family received genetic counselling for a presumed autosomal recessive monogenic disorder. A further four siblings are healthy.

The parents declined invasive prenatal genetic testing following identification of microcephaly and suspected pontocerebellar hypoplasia in the present case. Following an emergency Caesarean section for suspected fetal distress, the proband was intubated in the delivery room for poor respiratory effort and transferred to the neonatal intensive care unit. He remained ventilator-dependent with minimal respiratory effort. The proband had microcephaly with a head circumference $\mathrm{Z}$ score of -2.5 with otherwise normal growth parameters. He had abnormal movements with fisting, back-arching and posturing. There were no electrographic seizures. Neuroimaging with cranial ultrasound and later MRI demonstrated microcephaly with cerebellar hypoplasia (Figure 1B).

The proband was referred for clinical genetics assessment at age 9 hours and approved for inclusion in a rapid genomic diagnosis program, the Australian Genomics Acute Care study, at age 12 hours. Following genetic counselling, the parents provided written consent for trio exome sequencing. Chromosomal microarray was requested and performed in tandem with rapid exome sequencing (see Figure 1C).

Multiple homozygous variants were observed during variant prioritization, suggesting identity by descent despite absence of known parental consanguinity. Trio exome

This article is protected by copyright. All rights reserved. 
sequencing identified a homozygous splicing variant in ASNS

(Chr7(GRCh37):g.97482371C>T; NM_133436.3(ASNS):c.1476+1G>A). The essential splice-site variant was very highly conserved (PhyloP UCSC), absent from population databases (gnomAD, dbSNP, 1000G) and not previously reported in the ClinVar or HGMD databases or the medical literature (all databases accessed January 2019). Segregation analysis confirmed biparental inheritance. Biallelic mutations in ASNS cause asparagine synthetase deficiency (MIM\# 615574) (Alfadhel \& El-Hattab, 2018), a diagnosis consistent with the clinical features of the proband and his deceased sibling. The variant was classified as a variant of uncertain significance. Turnaround time from receipt of clinical specimens to issue of report was 75.5 hours.

The trio exome sequencing results were disclosed to the parents, and written consent was obtained for supportive analyses to determine the pathogenicity of the variant, including biochemical measurement of asparagine in blood and CSF, segregation of the variant in the deceased sibling from DNA extracted from a cryopreserved fibroblast cell line, and mRNA splicing analyses of blood obtained from the proband and both parents, and later from a cryopreserved fibroblast cell line from the deceased sibling.

Blood and CSF asparagine levels were $27 \mu \mathrm{mol} / \mathrm{L}$ (reference range 29-202) and 4 $\mu \mathrm{mol} / \mathrm{L}$ (reference range $0-20$ ), respectively. These values were considered potentially consistent with, but not diagnostic of, asparagine synthetase deficiency.

Chromosomal microarray results for the proband became available on day 9 , demonstrating no clinically significant genomic imbalance. Two regions of homozygosity were detected on chromosomes 7 and 8 . ASNS, located at chromosomal location 7q21.3, was within the chromosome 7 region of homozygosity. Retrospective This article is protected by copyright. All rights reserved. 
review of the deceased sibling's microarray performed in 2011 demonstrated a similar region of homozygosity on chromosome 7 that also included the ASNS gene (Supplementary Information, Figure S1).

Sanger sequencing of DNA extracted from a cryopreserved fibroblast cell line from a skin biopsy obtained from the deceased sibling identified the ASNS splicing variant in homozygous form.

Data mining of RNA-seq data obtained from the GTEx Project (Consortium, 2013) showed that ASNS exon 12 is a canonical exon included in predominant ASNS isoforms expressed in brain, blood and skin (Supplementary Information, Figure S2). Therefore, splicing outcomes observed in blood and fibroblast RNA maintain relevance to the predominant $A S N S$ isoform(s) in brain.

RT-PCR splicing studies of ASNS cDNA synthesized from mRNA isolated from whole blood (proband and both parents) and fibroblasts (deceased sibling) confirmed abnormal splicing induced by the homozygous $A S N S$ c. $1476+1 \mathrm{G}>\mathrm{A}$ variant (Figure 2). Abnormal splicing events included: a) exon 12 skipping which removes 156 nucleotides from the ASNS mRNA. This event is in-frame, removing 52 amino acids from the encoded asparagine synthetase enzymatic domain (p.(Asn441_Gln492del)); b) use of a cryptic 5'splice-site 48 nucleotides upstream of the native 5'splice-site which removes 48 nucleotides from exon 12. This event is in-frame, removing 16 amino acids from the asparagine synthetase enzymatic domain (p.(Val477_Gln492del)); c) retention of intron 12, or both intron 11 and intron 12, which encode a premature termination codon and may be targeted by nonsensemediated decay. Transcripts that may escape nonsense-mediated decay encode asparagine synthetase proteins that lack a conserved region within the asparagine

This article is protected by copyright. All rights reserved. 
synthetase enzymatic domain and are likely to be dysfunctional or non-functional (Figure 1D-E). A primer bridging exons 12 and 13, specific for normally-spliced ASNS transcripts, failed to amplify a product from cDNA for both affected siblings, though robustly amplified correctly spliced ASNS cDNA from the parent carriers and unrelated disease controls (Figure 2iii). These data infer there are no, or undetectably low levels of, correctly spliced ASNS transcripts in both affected individuals. Amplification of GAPDH at sub-saturating cycle lengths of 25 and 30 cycles confirmed similar cDNA loading for all samples.

The results of biochemical evaluation, chromosomal microarray, and mRNA splicing analyses of the proband and both parents (reported within 10 days) were available on day 27 of life. Based on the additional evidence, the homozygous ASNS splicing variant was reclassified as pathogenic according to ACMG guidelines (Richards et al., 2015), establishing a diagnosis of asparagine synthetase deficiency (MIM\# 615574) (Alfadhel \& El-Hattab, 2018).

Genetic counselling was provided to the family and after a further 13 days with their child, the parents elected to redirect care towards palliation and symptomatic management, and the proband died at age 40 days (Figure 1C)

Following receipt of segregation results in the deceased sibling, which became available subsequent to the proband's death, confirmatory mRNA splicing analyses were performed on cryopreserved fibroblasts from the deceased sibling (see Figure 2).

Total hospitalization length of stay for the proband and the deceased sibling was 40 days and 59 days respectively, with a difference of 19 days. Based on average daily hospitalization costs in a pediatric intensive care unit of AU\$6,200 (Schlapbach et al.,

This article is protected by copyright. All rights reserved. 
$2017)$, less the cost of ultra-rapid trio exome sequencing $(\$ 12,000)$ and research mRNA splicing analysis $(\$ 2,486$; reagents $\$ 433$, Sanger sequencing $\$ 1,363$, time $\$ 690$ (in silico analyses, laboratory work, reporting)), this difference in length of stay equates to an estimated cost-saving following early diagnosis of AU\$100,828. Potential additional cost savings related to reproductive testing to avoid another recurrence have not been estimated.

Rapid genomic diagnosis programs have the potential to target therapy, clarify prognosis, avoid unnecessary or invasive investigations and interventions, and reduce health costs (Farnaes et al., 2018; French et al., 2019; Meng et al., 2017; MestekBoukhibar et al., 2018; Petrikin et al., 2018; Stark et al., 2018; van Diemen et al., 2017; Willig et al., 2015). Variants of uncertain significance are frequently identified by genomic testing (Grody, Thompson, \& Hudgins, 2013). In the acute care setting, time taken to achieve diagnostic certainty may have a significant impact on clinical care. Despite recognition of the challenges associated with variants of uncertain significance (Petrikin, Willig, Smith, \& Kingsmore, 2015), supportive functional genomics analyses have not been described to date as part of a rapid genomic diagnosis program (Farnaes et al., 2018; French et al., 2019; Meng et al., 2017; Mestek-Boukhibar et al., 2018; Petrikin et al., 2018; Stark et al., 2018; van Diemen et al., 2017; Willig et al., 2015). With a molecular diagnosis of a rare monogenic disorder achieved following mRNA analyses within the first four weeks of life, this case demonstrates the clinical and cost benefits of incorporating supportive testing as an adjunct to rapid genomic diagnosis programs. Results facilitated clinical decision making within a clinically appropriate timeframe, resulting in redirection of care towards palliation and symptomatic management, with projected cost savings by reducing hospitalization length of stay and diagnostic investigations following early This article is protected by copyright. All rights reserved. 
diagnosis. Importantly, a confirmed diagnosis of asparagine synthetase deficiency now enables reproductive genetic testing.

Asparagine synthetase deficiency is a rare monogenic condition characterized by microcephaly with a progressive encephalopathy, severely delayed neurodevelopment, and early death (Alfadhel \& El-Hattab, 2018). The spectrum of clinical severity varies between reported cases (Abhyankar et al., 2018; Alfadhel et al., 2015; Alrifai \& Alfadhel, 2016; Ben-Salem et al., 2015; Galada et al., 2018; Gupta et al., 2017; Palmer et al., 2015; Sacharow et al., 2018; Seidahmed et al., 2016; Sun et al., 2017; Yamamoto et al., 2017), with no reported genotype-phenotype correlations (Alfadhel \& El-Hattab, 2018). It has been suggested that asparagine synthetase deficiency should be considered in any neonate with microcephaly and an epileptic encephalopathy, which is the most common clinical presentation for this condition (Radha Rama Devi \& Naushad, 2019). The two siblings in this report presented with a severe phenotype, with cerebellar hypoplasia and ventilator dependence from birth. mRNA analyses suggested absence, or extremely low levels, of normal ASNS splicing, undetectable by PCR amplification. Two of the three abnormal splicing events maintained the asparagine synthetase open reading frame, though deleted multiple sequential evolutionarily conserved amino acids that comprise the enzymatic domain (deleted residues are conserved to yeast/zebrafish). The severe neurological phenotype observed in both affected siblings is consistent with the high likelihood of enzymatic dysfunction or protein deficiency resulting from abnormal splicing of $A S N S$ transcripts, which affects the conserved asparagine synthetase enzymatic domain. Although emerging technologies in clinical genetics raise the exciting potential for targeted treatments, therapies with asparagine have had

This article is protected by copyright. All rights reserved. 
mixed results (Alrifai \& Alfadhel, 2016; Sprute et al., 2019) and the degree of brain malformation makes the success of postnatal intervention less likely.

Subsequent to diagnosis, RNA derived from blood (proband) and fibroblasts (deceased sibling) was subject to short-read RNA sequencing (150 nt paired-end reads). The blood specimen failed. RNA sequencing of the fibroblasts showed clear evidence for mis-splicing (exon-12 skipping, intron-12 retention, 5'cryptic splice site use). However, $15 \%$ of reads with cryptic splice site use were mis-mapped (100/667) and incorrectly aligned to the authentic 5'splice site junction with mis-matching (Supplementary Information, Figure S3). Short read RNA sequencing is prone to mismapping, and particularly for mis-spliced reads that do not match the reference transcriptome. Short-read RNA sequencing is powerful though holds inherent limitations in that reads do not bridge multiple exons, in some cases presenting diagnostic uncertainty related to which isoform(s) and which allele(s) is/are affected by mis-splicing, especially in cases with alternative splicing of exons adjacent to a putative splicing variant. Due to these considerations, we recommend abnormal findings observed by short read RNA sequencing be confirmed by RT-PCR before being used clinically. Long read sequencing is entering the diagnostic horizon and may hold improved time and cost-efficiencies for diagnostic use as an alternative to multiple, bespoke PCRs, gel extraction and Sanger sequencing of amplicons.

In conclusion, we present the neonatal diagnosis of asparagine synthetase deficiency facilitated by rapid genomic testing (exome sequencing and adjunct mRNA analyses) of a critically unwell neonate. Whilst not all variants of uncertain significance are amenable to clinical functional genomics, establishment of a rapid pipeline for mRNA

This article is protected by copyright. All rights reserved. 
analyses has potential to increase the number of definitive diagnoses, with significant clinical and health economic benefits.

\section{ACKNOWLEDGEMENTS}

We thank the family for their willingness to participate in this research study, in this time of great stress and sadness. We also acknowledge the contributions of the clinicians and health care workers who managed their care.

\section{DATA AVAILABILITY STATEMENT}

The data reported in this case report have not been submitted to a publicly available database to respect patient confidentiality.

\section{REFERENCES}

Abhyankar, A., Lamendola-Essel, M., Brennan, K., Giordano, J. L., Esteves, C., Felice, V.,... Jobanputra, V. (2018). Clinical whole exome sequencing from dried blood spot identifies novel genetic defect underlying asparagine synthetase deficiency. Clin Case Rep, 6(1), 200-205. doi:10.1002/ccr3.1284

Alfadhel, M., Alrifai, M. T., Trujillano, D., Alshaalan, H., Al Othaim, A., Al Rasheed, S.,... Eyaid, W. (2015). Asparagine Synthetase Deficiency: New Inborn Errors of Metabolism. JIMD Rep, 22, 11-16. doi:10.1007/8904_2014_405

Alfadhel, M., \& El-Hattab, A. W. (2018). Asparagine Synthetase Deficiency. In M. P. Adam, H. H. Ardinger, R. A. Pagon, S. E. Wallace, L. J. H. Bean, K. Stephens, \& A. Amemiya (Eds.), GeneReviews((R)). Seattle (WA).

This article is protected by copyright. All rights reserved. 
Alrifai, M. T., \& Alfadhel, M. (2016). Worsening of Seizures After Asparagine Supplementation in a Child with Asparagine Synthetase Deficiency. Pediatr Neurol, 58, 98-100. doi:10.1016/j.pediatrneurol.2016.01.024

Ben-Salem, S., Gleeson, J. G., Al-Shamsi, A. M., Islam, B., Hertecant, J., Ali, B. R., \& AlGazali, L. (2015). Asparagine synthetase deficiency detected by whole exome sequencing causes congenital microcephaly, epileptic encephalopathy and psychomotor delay. Metab Brain Dis, 30(3), 687-694. doi:10.1007/s11011-014-9618-0 Clark, M. M., Hildreth, A., Batalov, S., Ding, Y., Chowdhury, S., Watkins, K.,... Kingsmore, S. F. (2019). Diagnosis of genetic diseases in seriously ill children by rapid whole-genome sequencing and automated phenotyping and interpretation. Sci Transl Med, 11(489). doi:10.1126/scitranslmed.aat6177

Consortium, G. T. (2013). The Genotype-Tissue Expression (GTEx) project. Nat Genet, 45(6), 580-585. doi:10.1038/ng.2653

Farnaes, L., Hildreth, A., Sweeney, N. M., Clark, M. M., Chowdhury, S., Nahas, S.,... Kingsmore, S. F. (2018). Rapid whole-genome sequencing decreases infant morbidity and cost of hospitalization. NPJ Genom Med, 3, 10. doi:10.1038/s41525-018-0049-4

French, C. E., Delon, I., Dolling, H., Sanchis-Juan, A., Shamardina, O., Megy, K.,... Raymond, F. L. (2019). Whole genome sequencing reveals that genetic conditions are frequent in intensively ill children. Intensive Care Med, 45(5), 627-636. doi:10.1007/s00134-019-05552-x 
Galada, C., Hebbar, M., Lewis, L., Soans, S., Kadavigere, R., Srivastava, A.,... Shukla, A. (2018). Report of four novel variants in ASNS causing asparagine synthetase deficiency and review of literature. Congenit Anom (Kyoto), 58(5), 181-182. doi:10.1111/cga.12275

Grody, W. W., Thompson, B. H., \& Hudgins, L. (2013). Whole-exome/genome sequencing and genomics. Pediatrics, 132(Suppl 3), S211-215.

doi:10.1542/peds.2013-1032E

Gupta, N., Tewari, V. V., Kumar, M., Langeh, N., Gupta, A., Mishra, P.,... Kabra, M. (2017). Asparagine Synthetase deficiency-report of a novel mutation and review of literature. Metab Brain Dis, 32(6), 1889-1900. doi:10.1007/s11011-017-0073-6

Meng, L., Pammi, M., Saronwala, A., Magoulas, P., Ghazi, A. R., Vetrini, F.,... Lalani, S. R. (2017). Use of Exome Sequencing for Infants in Intensive Care Units: Ascertainment of Severe Single-Gene Disorders and Effect on Medical Management. JAMA Pediatr, 171(12), e173438. doi:10.1001/jamapediatrics.2017.3438

Mestek-Boukhibar, L., Clement, E., Jones, W. D., Drury, S., Ocaka, L., Gagunashvili, A.,... Williams, H. J. (2018). Rapid Paediatric Sequencing (RaPS): comprehensive reallife workflow for rapid diagnosis of critically ill children. J Med Genet, 55(11), 721-728. doi:10.1136/jmedgenet-2018-105396

Palmer, E. E., Hayner, J., Sachdev, R., Cardamone, M., Kandula, T., Morris, P.,... Kirk, E. P. (2015). Asparagine Synthetase Deficiency causes reduced proliferation of cells under conditions of limited asparagine. Mol Genet Metab, 116(3), 178-186. doi:10.1016/j.ymgme.2015.08.007

This article is protected by copyright. All rights reserved. 
Petrikin, J. E., Cakici, J. A., Clark, M. M., Willig, L. K., Sweeney, N. M., Farrow, E. G.,... Kingsmore, S. F. (2018). The NSIGHT1-randomized controlled trial: rapid wholegenome sequencing for accelerated etiologic diagnosis in critically ill infants. NPJ Genom Med, 3, 6. doi:10.1038/s41525-018-0045-8

Petrikin, J. E., Willig, L. K., Smith, L. D., \& Kingsmore, S. F. (2015). Rapid whole genome sequencing and precision neonatology. Semin Perinatol, 39(8), 623-631. doi:10.1053/j.semperi.2015.09.009

Radha Rama Devi, A., \& Naushad, S. M. (2019). Molecular diagnosis of asparagine synthetase (ASNS) deficiency in two Indian families and literature review of 29 ASNS deficient cases. Gene, 704, 97-102. doi:10.1016/j.gene.2019.04.024

Richards, S., Aziz, N., Bale, S., Bick, D., Das, S., Gastier-Foster, J.,... Committee, A. L. Q. A. (2015). Standards and guidelines for the interpretation of sequence variants: a joint consensus recommendation of the American College of Medical Genetics and Genomics and the Association for Molecular Pathology. Genet Med, 17(5), 405-424. doi:10.1038/gim.2015.30

Sacharow, S. J., Dudenhausen, E. E., Lomelino, C. L., Rodan, L., El Achkar, C. M., Olson, H. E.,... Kilberg, M. S. (2018). Characterization of a novel variant in siblings with Asparagine Synthetase Deficiency. Mol Genet Metab, 123(3), 317-325. doi:10.1016/j.ymgme.2017.12.433

Schlapbach, L. J., Straney, L., Gelbart, B., Alexander, J., Franklin, D., Beca, J.,... New Zealand Intensive Care Society Paediatric Study, G. (2017). Burden of disease and 
change in practice in critically ill infants with bronchiolitis. Eur Respir J, 49(6). doi:10.1183/13993003.01648-2016

Seidahmed, M. Z., Salih, M. A., Abdulbasit, O. B., Samadi, A., Al Hussien, K., Miqdad, A. M.,... Alkuraya, F. S. (2016). Hyperekplexia, microcephaly and simplified gyral pattern caused by novel ASNS mutations, case report. BMC Neurol, 16, 105. doi:10.1186/s12883-016-0633-0

Sprute, R., Ardicli, D., Oguz, K. K., Malenica-Mandel, A., Daimaguler, H. S., Koy, A.,... Cirak, S. (2019). Clinical outcomes of two patients with a novel pathogenic variant in ASNS: response to asparagine supplementation and review of the literature. Hum Genome Var, 6, 24. doi:10.1038/s41439-019-0055-9

Stark, Z., Lunke, S., Brett, G. R., Tan, N. B., Stapleton, R., Kumble, S.,... Melbourne Genomics Health, A. (2018). Meeting the challenges of implementing rapid genomic testing in acute pediatric care. Genet Med, 20(12), 1554-1563.

doi:10.1038/gim.2018.37

Sun, J., McGillivray, A. J., Pinner, J., Yan, Z., Liu, F., Bratkovic, D.,... Chopra, M. (2017). Diaphragmatic Eventration in Sisters with Asparagine Synthetase Deficiency: A Novel Homozygous ASNS Mutation and Expanded Phenotype. JIMD Rep, 34, 1-9. doi:10.1007/8904_2016_3

van Diemen, C. C., Kerstjens-Frederikse, W. S., Bergman, K. A., de Koning, T. J., Sikkema-Raddatz, B., van der Velde, J. K.,... Wijmenga, C. (2017). Rapid Targeted Genomics in Critically III Newborns. Pediatrics, 140(4), e20162854. doi:10.1542/peds.2016-2854

This article is protected by copyright. All rights reserved. 
Willig, L. K., Petrikin, J. E., Smith, L. D., Saunders, C. J., Thiffault, I., Miller, N. A.,... Kingsmore, S. F. (2015). Whole-genome sequencing for identification of Mendelian disorders in critically ill infants: a retrospective analysis of diagnostic and clinical findings. Lancet Respir Med, 3(5), 377-387. doi:10.1016/S2213-2600(15)00139-3 Yamamoto, T., Endo, W., Ohnishi, H., Kubota, K., Kawamoto, N., Inui, T.,... Fukao, T. (2017). The first report of Japanese patients with asparagine synthetase deficiency. Brain Dev, 39(3), 236-242. doi:10.1016/j.braindev.2016.09.010

\section{FIGURES}

Figure 1. Family pedigree, brain imaging, clinical time-line and schematic overview of pathogenic $A S N S$ variants. A) Magnetic resonance imaging (MRI) of postnatal brain for the proband showing cerebellar hypoplasia. B) Pedigree showing the proband (arrow) and his deceased affected sibling. C) Timeline of clinical and genetic investigations, noting sequential re-classification of the homozygous ASNS c. $1476+1 \mathrm{G}>\mathrm{A}$ variant from a variant of uncertain significance to a pathogenic variant, and subsequent changes in clinical management. Wks: weeks of age. D) A schematic of the encoded asparagine synthetase protein showing the position of residues deleted through abnormal splicing induced by the c. $1476+1 \mathrm{G}>\mathrm{A}$ variant, as well as positions of previously reported $A S N S$ variants classified as likely pathogenic or pathogenic taken from ClinVar. Residues annotated as comprising the functional domains are

This article is protected by copyright. All rights reserved. 
taken from Uniprot (P08243). E) Evolutionary alignment of the amino acid residues encoded by ASNS exon 12 (NM_001673.4). Skipping of Exon -12, or use of the upstream 5' cryptic splice site in exon 12 , abnormally removes numerous highly conserved amino acids, many of which are invariant throughout vertebrate evolution.

A

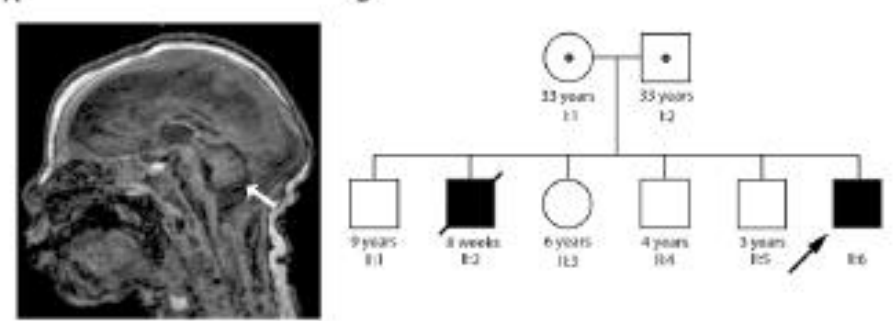

c

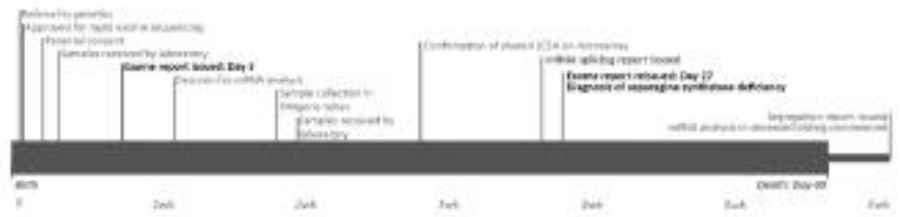

D

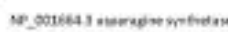

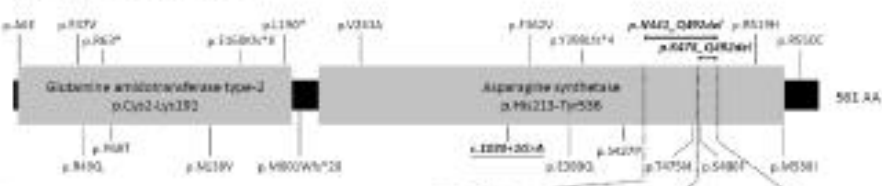

E

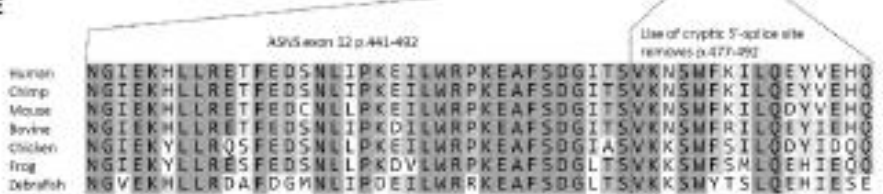

This article is protected by copyright. All rights reserved. 
Figure 2. Adjunct RT-PCR studies of ASNS pre-mRNA splicing. A) Gel

electrophoresis of reverse transcription polymerase chain reaction (RT-PCR) reactions. RT-PCR was performed using mRNA isolated from whole blood from the family trio and three controls $\left(\mathrm{C}_{1}=\right.$ disease control 1 , male, 7 months; $\mathrm{C}_{2}=$ disease control 2 , male, 5 years; $\mathrm{C}_{3}=$ control 3 , female, 43 years), and subsequently, using cDNA from primary fibroblasts from the affected sibling and two controls $\left(\mathrm{C}_{4}=\right.$ disease control 4, Male, 8 months; $\mathrm{C}_{5}=$ control 5, male fetus 31/40 weeks). $\mathrm{P}=$ affected proband; $\mathrm{M}=$ unaffected mother; $\mathrm{F}=$ unaffected father; $\mathrm{S}=$ affected sibling. i) Forward primer in exon 10 and reverse primer in exon 13. ii) Forward primer in exon 12 and reverse primer in the 3'UTR. iii) Forward primer bridging the junction of exon-12/ exon-13 to selectively amplify transcripts with normal splicing of exon 1213. We could not find evidence for normal exon 12-13 splicing in the proband and his sibling. $i v$ ) Forward primer in exon 10 and reverse primer in intron 12 to selectively amplify transcripts with intron 12 retention. Retention of intron 12, and or intron 11 and 12 , was not detected in controls and observed only in the affected individuals and parent carriers of the c. $1476+1 \mathrm{G}>\mathrm{A}$ variant. $v$ ) Forward and reverse primers in GAPDH used as a cDNA loading control. Replicate samples were subject to PCR for 25 or 30 cycles to confirm sub-saturating PCR conditions and demonstrate loading and quality of cDNA. B) Sanger Sequencing of gel purified bands. Amplicon 1 was confirmed to correspond to normal splicing of exons 10-11-12-13 (444 bp). Amplicon 2 corresponds to use of an exon 12 cryptic 5' splice site (396 bp), 48 nucleotides upstream from the authentic 5' splice site. Amplicon 3 corresponds to exon-12 skipping (288 bp). Amplicon 4 corresponds to retention of intron 12 (322 bp). Amplicon 5 corresponds to retention of both intron 11 and 12 (411 bp). C) Schematic of the abnormal splicing events induced by the ASNS c. $1476+1 \mathrm{G}>\mathrm{A}$ variant, showing

This article is protected by copyright. All rights reserved. 
the positions of the exon $125^{\prime}$ cryptic splice site and encoded stop codons resulting from retention of intron 11 or intron 12 .

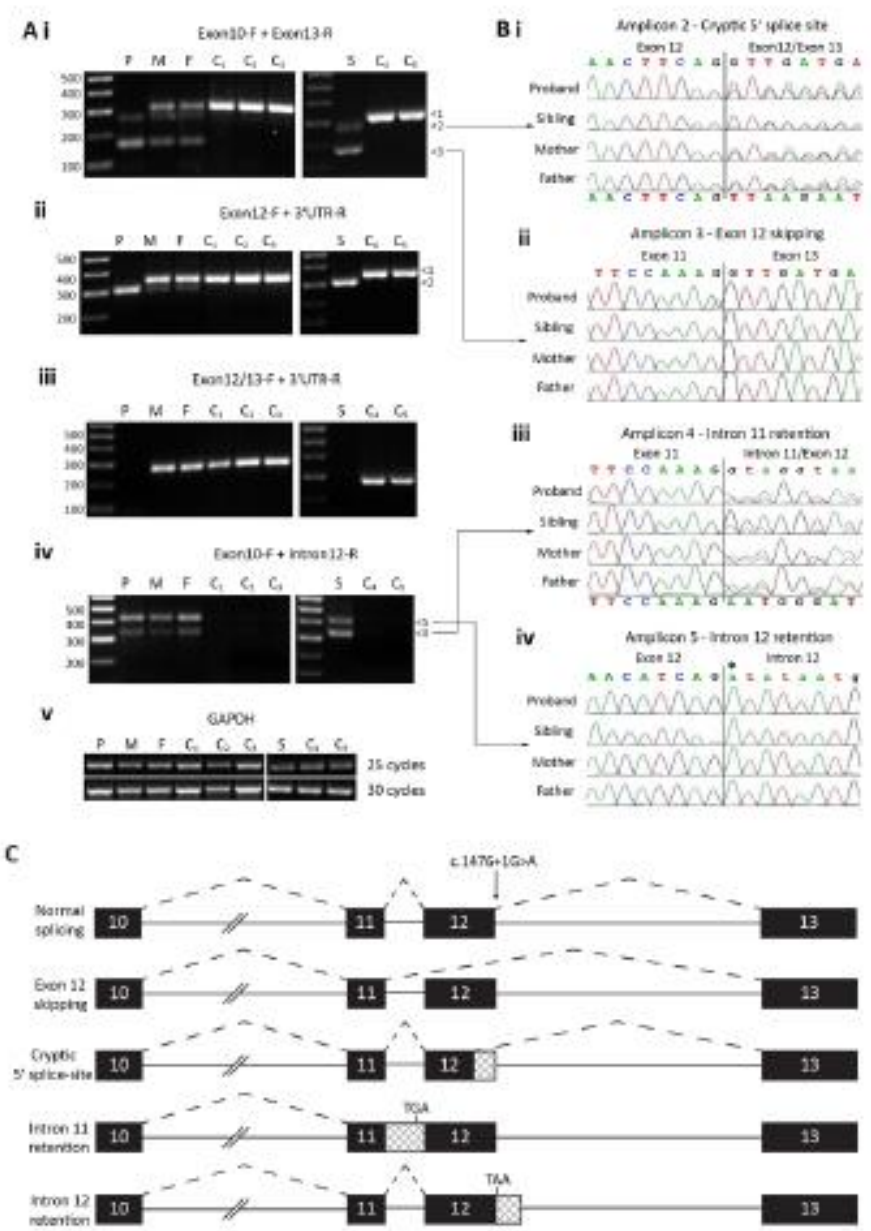

This article is protected by copyright. All rights reserved. 


\section{University Library}

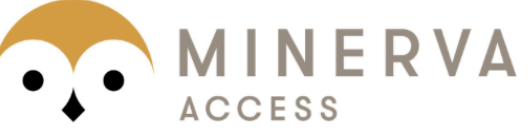

A gateway to Melbourne's research publications

Minerva Access is the Institutional Repository of The University of Melbourne

\section{Author/s:}

Akesson, LS;Bournazos, A;Fennell, A;Krzesinski, El;Tan, K;Springer, A;Rose, K;Goranitis, I;Francis, D;Lee, C;Faiz, F;Davis, MR;Christodoulou, J;Lunke, S;Stark, Z;Hunter, MF;Cooper, ST

Title:

Rapid exome sequencing and adjunct RNA studies confirm the pathogenicity of a novel homozygousASNSsplicing variant in a critically ill neonate

\section{Date:}

2020-11

\section{Citation:}

Akesson, L. S., Bournazos, A., Fennell, A., Krzesinski, E. I., Tan, K., Springer, A., Rose, K., Goranitis, I., Francis, D., Lee, C., Faiz, F., Davis, M. R., Christodoulou, J., Lunke, S., Stark, Z., Hunter, M. F. \& Cooper, S. T. (2020). Rapid exome sequencing and adjunct RNA studies confirm the pathogenicity of a novel homozygousASNSsplicing variant in a critically ill neonate. HUMAN MUTATION, 41 (11), pp.1884-1891. https://doi.org/10.1002/humu.24101.

Persistent Link:

http://hdl.handle.net/11343/276249 